\title{
Dichloroacetate as a possible treatment for endometriosis-associated pain: a single-arm open-label exploratory clinical trial (EPiC)
}

H. W. Leow ${ }^{1}$, M. Koscielniak', L. Williams², P. T. K. Saunders ${ }^{3}$, J. Daniels ${ }^{4}$, A. M. Doust ${ }^{1}$, M-C Jones ${ }^{5}$, G. D. Ferguson ${ }^{6}$, Y. Bagger ${ }^{6}$, A. W. Horne ${ }^{1 *}$ (D) and L. H. R. Whitaker ${ }^{1}$

\begin{abstract}
Background: Endometriosis (where endometrial-like tissue is found outside the uterus) affects $\sim 176$ million women worldwide and can lead to debilitating pelvic pain. There is an unmet need for new medical treatment options for endometriosis. Pelvic peritoneal mesothelial cells of women with endometriosis exhibit detrimental metabolic reprogramming that creates an environment favouring the formation and survival of endometriosis lesions. We have generated powerful preclinical proof-of-concept data to show that it is possible to correct this metabolic phenotype using dichloroacetate (DCA), a non-hormonal compound previously used to treat rare metabolic disorders in children. We plan a single-arm, open-label, single site exploratory clinical trial to inform the design of a future randomised controlled trial (RCT) to determine the efficacy of DCA for the treatment of endometriosis-associated pain.
\end{abstract}

Methods: We will recruit 30 women with endometriosis-associated pain over a 6-month period. All participants will receive approximately $6.25 \mathrm{mg} / \mathrm{kg}$ oral DCA capsules twice daily for 6 weeks, with a dose increase to approximately $12.5 \mathrm{mg} / \mathrm{kg}$ twice daily for a further 6 weeks if their pain has not been adequately controlled on this dose regime and side-effects are acceptable. If pain is adequately controlled with minimal side-effects, the lower dose will be continued for a further 6 weeks. The primary objective is to determine whether it is possible to achieve acceptable recruitment and retention rates within the defined exclusion and inclusion criteria. Secondary objectives are to determine the acceptability of the trial to participants, including the proposed methods of recruitment, treatment, follow-up frequency and number of questionnaires. The recruitment rate will be determined by the proportion of patients recruited from the pool of eligible women. The retention rate will be determined by the proportion of participants who attended the final trial visit.

Discussion: This is a feasibility study to explore effectiveness and acceptability of the proposed field methodology (recruitment, retention, study processes and compliance with treatment). The results will be used to inform the design of a future RCT.

Trial registration: ClinicalTrials.gov, NCT04046081 Registered 6 August 2019

Keywords: Chronic pelvic pain, Gynaecology, Glycolysis, Repurposing, Feasibility trial

\footnotetext{
* Correspondence: Andrew.horne@ed.ac.uk

'MRC Centre for Reproductive Health, Queen's Medical Research Institute, University of Edinburgh, 47 Little France Crescent, Edinburgh EH16 4TJ, UK

Full list of author information is available at the end of the article
}

C C The Author(s). 2021 Open Access This article is licensed under a Creative Commons Attribution 4.0 International License, which permits use, sharing, adaptation, distribution and reproduction in any medium or format, as long as you give appropriate credit to the original author(s) and the source, provide a link to the Creative Commons licence, and indicate if changes were made. The images or other third party material in this article are included in the article's Creative Commons licence, unless indicated otherwise in a credit line to the material. If material is not included in the article's Creative Commons licence and your intended use is not permitted by statutory regulation or exceeds the permitted use, you will need to obtain permission directly from the copyright holder. To view a copy of this licence, visit http://creativecommons.org/licenses/by/4.0/ The Creative Commons Public Domain Dedication waiver (http://creativecommons.org/publicdomain/zero/1.0/) applies to the data made available in this article, unless otherwise stated in a credit line to the data. 


\section{Background}

Endometriosis is characterised by the presence of endometrial-like tissue outside the uterus, commonly on the lining of the pelvic cavity [1]. It is a chronic, inflammatory gynaecological condition that affects approximately $10 \%$ of women of reproductive age [2]. It is associated with debilitating pelvic pain, pain during menstruation, pain during intercourse and subfertility $[3,4]$. Direct healthcare costs amount to nearly 3000 Euros per woman per year (based on 2009 prices), similar to those of diabetes mellitus [5]. Current treatment options are often unsatisfactory. Of women undergoing surgery, over half would have a further surgical procedure by 5 years [6]. Available hormonal treatments have unwanted side-effects and are contraceptive [7]. There is an 'unmet need for new medical treatments for endometriosis' which are disease modifying and fertility sparing [8].

Endometriosis exhibits cancer-like features [9]. Specifically, tumour cells are programmed by TGF- $\beta 1$ to use aerobic glycolysis resulting in increased secretion of lactate $[10-12]$. We have shown that TGF- $\beta 1$ and lactate are both elevated in the peritoneal fluid of women with endometriosis and that this is paralleled by a switch from normal mitochondrial respiration towards glycolysis in the human peritoneal mesothelial cells (HPMC) that line the pelvic cavity [10-12]. In the tumour microenvironment, lactate is considered a key factor in driving cell invasion, angiogenesis and immune suppression [13], all of which are implicated in the establishment and survival of endometriosis lesions. Using in vitro models, we have demonstrated that the glycolysis inhibitor, dichloroacetate (DCA), reversed the aberrantly increased glycolysis of HPMC and oral administration of DCA reduced the size of endometriosis lesions in a mouse model [14].

We plan a single-arm open-label exploratory clinical trial to determine the feasibility of achieving an acceptable recruitment and retention rates. This trial will inform the design of a potential future large randomised controlled trial (RCT) to determine the efficacy of DCA to treat women with endometriosis-associated pain.

\section{Methods/design}

\section{Primary outcome}

To determine whether it is possible to achieve acceptable recruitment and retention rates within the defined inclusion/exclusion criteria

\section{Secondary outcomes}

- To determine the acceptability to participants of the proposed method of recruitment, treatment, questionnaires and follow-up (e.g. frequency and method for collecting pain scores and for completion of questionnaires)

- To assess how well-tolerated is DCA in women with endometriosis

- To determine if we can detect systemic DCA in blood samples of women with endometriosis

- To determine participants' compliance with treatment and to assess the tools used to measure compliance

\section{Study design}

This is a single site, open-label exploratory study of women with endometriosis.

\section{Study population}

We will recruit 30 women with endometriosis-associated pain over a 6-month period. The duration of the entire trial will be 12 months. The trial visits will take place within NHS Lothian, UK. The participants will be required to come to the hospital for five visits, at day 1 and at weeks 2, 6, 8 and 12 to complete questionnaires, give blood samples and receive further DCA supplies if required. The participants will be taking the treatment for 12 weeks and will receive a phone call 4 weeks $( \pm 1$ week) after the last date of treatment to ask about their general health and how satisfied they are with the treatment and trial methods (see Table 1).

\section{Inclusion criteria}

- Pre-menopausal women aged 18 or over

- Weight between 50 and $100 \mathrm{~kg}$

- Superficial peritoneal endometriosis (ASRM stage I or II) identified at laparoscopy, performed within the last five years (and > 2 weeks from surgery)

- Pelvic pain for longer than 6 months

- Average pain score of $\geq 4$ over the 4 weeks prior to treatment

- Willing to comply with the treatment

- Willing to use non-hormonal contraception throughout the trial

- Willing and able to complete informed consent

\section{Exclusion criteria}

- Evidence of ovarian endometrioma or deep endometriosis (based upon current surgical staging or most recent imaging)

- Women who are pregnant or actively trying to get pregnant

- Known allergy or hypersensitivity to any excipient of DCA 
Table 1 Summary of study visits and assessments

\begin{tabular}{|c|c|c|c|c|c|c|c|c|c|c|c|c|c|c|c|}
\hline Phase & Baseline & \multicolumn{12}{|c|}{ Treatment week } & End of & Follow-up \\
\hline \multirow{2}{*}{ Duration (weeks) } & \multirow{2}{*}{ Day 1} & \multicolumn{6}{|c|}{$1-6$} & \multicolumn{6}{|c|}{$7-12$} & \multirow{2}{*}{$\begin{array}{c}\text { End of } \\
\text { week } 12\end{array}$} & \multirow{2}{*}{16} \\
\hline & & 1 & 2 & 3 & 4 & 5 & 6 & 7 & 8 & 9 & 10 & 11 & 12 & & \\
\hline Consent & $\mathbf{x}$ & & & & & & & & & & & & & & \\
\hline $\begin{array}{l}\text { Inclusion/ exclusion } \\
\text { eligibility }\end{array}$ & $\mathbf{x}$ & & & & & & & & & & & & & & \\
\hline $\begin{array}{l}\text { Medical History } \\
\text { Pregnancy test }\end{array}$ & $\mathbf{x}$ & & & & & & & & & & & & & & \\
\hline $\begin{array}{l}\text { Recalled 'average' NRS } \\
\text { over previous } 4 \text { weeks }\end{array}$ & $\mathbf{x}$ & & & & & & $\mathbf{x}$ & & & & & & & $\mathbf{x}$ & \\
\hline \multicolumn{16}{|l|}{ Questionnaires } \\
\hline $\begin{array}{l}\text { Endometriosis Health } \\
\text { Profile-30 }\end{array}$ & $\mathbf{x}$ & & & & & & $\mathbf{x}$ & & & & & & & $\mathbf{x}$ & \\
\hline PainDetect ${ }^{\mathrm{TM}}$ & $\mathbf{x}$ & & & & & & $\mathbf{x}$ & & & & & & & $\mathbf{x}$ & \\
\hline $\begin{array}{l}\text { Brief Fatigue Inventory } \\
\text { (BFI) }\end{array}$ & $\mathbf{x}$ & & & & & & $\mathbf{x}$ & & & & & & & $\mathbf{x}$ & \\
\hline $\begin{array}{l}\text { Pain Catastrophising } \\
\text { Questionnaire }\end{array}$ & $\mathbf{x}$ & & & & & & $\mathbf{x}$ & & & & & & & $\mathbf{x}$ & \\
\hline $\begin{array}{l}\text { Treatment and trial } \\
\text { acceptability questionnaire }\end{array}$ & & & & & & & & & & & & & & & $\mathbf{x}$ \\
\hline \multicolumn{16}{|l|}{ Assessments } \\
\hline Blood samples & $\mathbf{x}$ & & $\mathbf{x}$ & & & & $\mathbf{x}$ & & $\mathbf{x}$ & & & & & $\mathbf{x}$ & \\
\hline Side effects & & & $\mathbf{x}$ & & & & $\mathbf{x}$ & & $\mathbf{x}$ & & & & & $\mathbf{x}$ & $\mathbf{x}$ \\
\hline $\begin{array}{l}\text { Permitted / Concomitant } \\
\text { medication }\end{array}$ & $\mathbf{x}$ & & $\mathbf{x}$ & & & & $\mathbf{x}$ & & $\mathbf{x}$ & & & & & $\mathbf{x}$ & \\
\hline $\begin{array}{l}\text { Adherence or } \\
\text { discontinuation }\end{array}$ & & & $\mathbf{x}$ & & & & $\mathbf{x}$ & & $\mathbf{x}$ & & & & & $\mathbf{x}$ & \\
\hline \multicolumn{16}{|l|}{$\begin{array}{l}\text { Dose change (if } \\
\text { acceptable) }\end{array}$} \\
\hline Dispense/resupply DCA & $\mathbf{x}$ & & $\mathbf{x}$ & & & & $\mathbf{x}$ & & $\mathbf{x}$ & & & & & & \\
\hline Return of unused DCA & & & & & & & $\mathbf{x}$ & & & & & & & $\mathbf{x}$ & \\
\hline
\end{tabular}

- Breastfeeding

- Clinical evidence of pre-existing peripheral neuropathy

- Diabetes, history of liver, or history of kidney disease

- Taking part in another Clinical Trial of an Investigational Medicinal Product (CTIMP) or other interventional non-CTIMP studies

- Patient on combination anti-retroviral therapy

- History of malabsorption syndrome or substantial amount of small bowel or stomach removed

\section{Participant enrolment}

Potentially eligible women will be asked by their attending clinician if they would be interested in participating in the study. If they agree, the women will then be given a patient information sheet and will be referred to members of the clinical research team to discuss the study in more detail. Patients will be allowed to self-refer to the study by contacting the research team directly. Informed consent will only be taken by a member of the research team once the participants have had ample time (at least $24 \mathrm{~h}$ ) to read the patient information sheet and had their 
questions answered. Following consent, eligibility will be assessed by the clinical team. A screening log will be maintained and the following anonymised information will be monitored and collected for all potential participants: date of attendance in clinic, year of birth, reason for non-eligibility and reason for not participating if eligible and willing to give a reason. Ineligible and nonrecruited participants will be offered routine NHS gynaecological care.

\section{Intervention}

Based on our current preclinical studies and the current literature, we have chosen a dose which will potentially be effective for endometriosis-associated pain and has the least side-effects. Trials using DCA as a potential treatment for cancer or pulmonary arterial hypertension suggest that doses of $6.25 \mathrm{mg} / \mathrm{kg}$ BD should produce blood levels within the range required for pyruvate dehydrogenase kinase (PDK) inhibition, although DCA pharmacokinetics may be variable [15]. We also note that most side-effects, such as reversible peripheral neuropathy, seem to occur at doses of $25-50 \mathrm{mg} / \mathrm{kg} /$ day [16-18]. We therefore plan to trial the feasibility of a dose change with DCA at approximately $6.25 \mathrm{mg} / \mathrm{kg}$ BD (oral) for 6 weeks, followed by a dose increase to approximately $12.5 \mathrm{mg} / \mathrm{kg}$ BD (oral) for the next 6 weeks, depending on participant's tolerance of treatment (total treatment phase of 12 weeks).

The drug will be provided in capsules containing 333 $\mathrm{mg}$ or $500 \mathrm{mg}$ of DCA powder (Curaltus Ltd, Lithuania). Participants will be required to take between two to six capsules orally a day depending on the dose calculated using their body weight. The drug will be dispensed on day 1 of treatment and resupplied if needed at each visit during week 2, 6 and 8. Sufficient amount of drug will be provided to last until the next visit or end of study.

1. Weeks 1-6. All participants will receive approximately $6.25 \mathrm{mg} / \mathrm{kg}$ BD daily (oral) for 6 weeks.

2. Weeks 6-12. Dosing may be escalated depending on symptom control and side-effects (options $1-3$ as below).

(a) Option 1. If the participant still has painful symptoms at the end of week 6 and minimal or no side-effects, she will be asked to increase the dose of DCA to approximately $12.5 \mathrm{mg} / \mathrm{kg} \mathrm{BD}$ daily (oral) for a further 6 weeks (see Figure 1, study flow chart). Dose escalation will be overseen by a medical doctor on the delegation log.

(b) Option 2. If DCA has been helpful in reducing painful symptoms and caused minimal sideeffects, then the participant will be asked to continue to take DCA $6.25 \mathrm{mg} / \mathrm{kg}$ BD daily

(oral) for a further 6 weeks.

(c) Option 3. If the participant suffers from sideeffects from DCA and the dose of DCA taken does not provide relief for endometriosis pain, the participant is allowed to stop taking the drug at any stage of the treatment, but they will continue to be followed up with their consent.

If participants who take $12.5 \mathrm{mg} / \mathrm{kg} \mathrm{BD}$ (oral) have side-effects and do not tolerate the treatment, they will be allowed to return to low dose of $6.25 \mathrm{mg} / \mathrm{kg} \mathrm{BD}$. If participants do not have enough capsules to return to low dose, they will be resupplied with new capsules.

\section{Assessment of participant compliance}

Participants will be asked about compliance at each visit, which will be recorded in the case report form (CRF). Compliance will be also measured via a treatment diary and systemic blood levels.

\section{Other medications}

Participants will be allowed to take any oral analgesics and alternative treatments (e.g. acupuncture) throughout the study period, which will be recorded in the CRF. Due to the potential for pain relief from hormonal therapy, patients will only be allowed to take hormonal therapies if they have started them more than three months before consent.

\section{Safety assessments}

At the first visit, a medical history will be taken to assess specific eligibility points, such as concurrent neuropathy, diabetes, liver or kidney disease, anti-retroviral use and use of concomitant medications. Contraception requirement and method will be documented and a pregnancy test undertaken. Participants will be assessed at each visit by a Good Clinical Practice (GCP)-trained medical doctor to confirm their wellbeing, including a neurological examination to assess for peripheral neuropathy.

\section{Study assessments}

Study visits and assessments are outlined in Table 1 . Stage of cycle will be documented at commencement of treatment.

Quality of Life and pain questionnaires include the Endometriosis Health Profile-30 [19], PainDETECT ${ }^{\mathrm{TM}}$ [20], Brief Fatigue Inventory (BFI) [21] and Pain Catastrophising Questionnaire [22]. At the end of the study (week 16) participants will be asked to complete an acceptability questionnaire, using a five point Likert scale. This consists of 11 questions to record acceptability of the proposed methods of recruitment, follow-up, treatments and use of quality of life and pain questionnaires. 


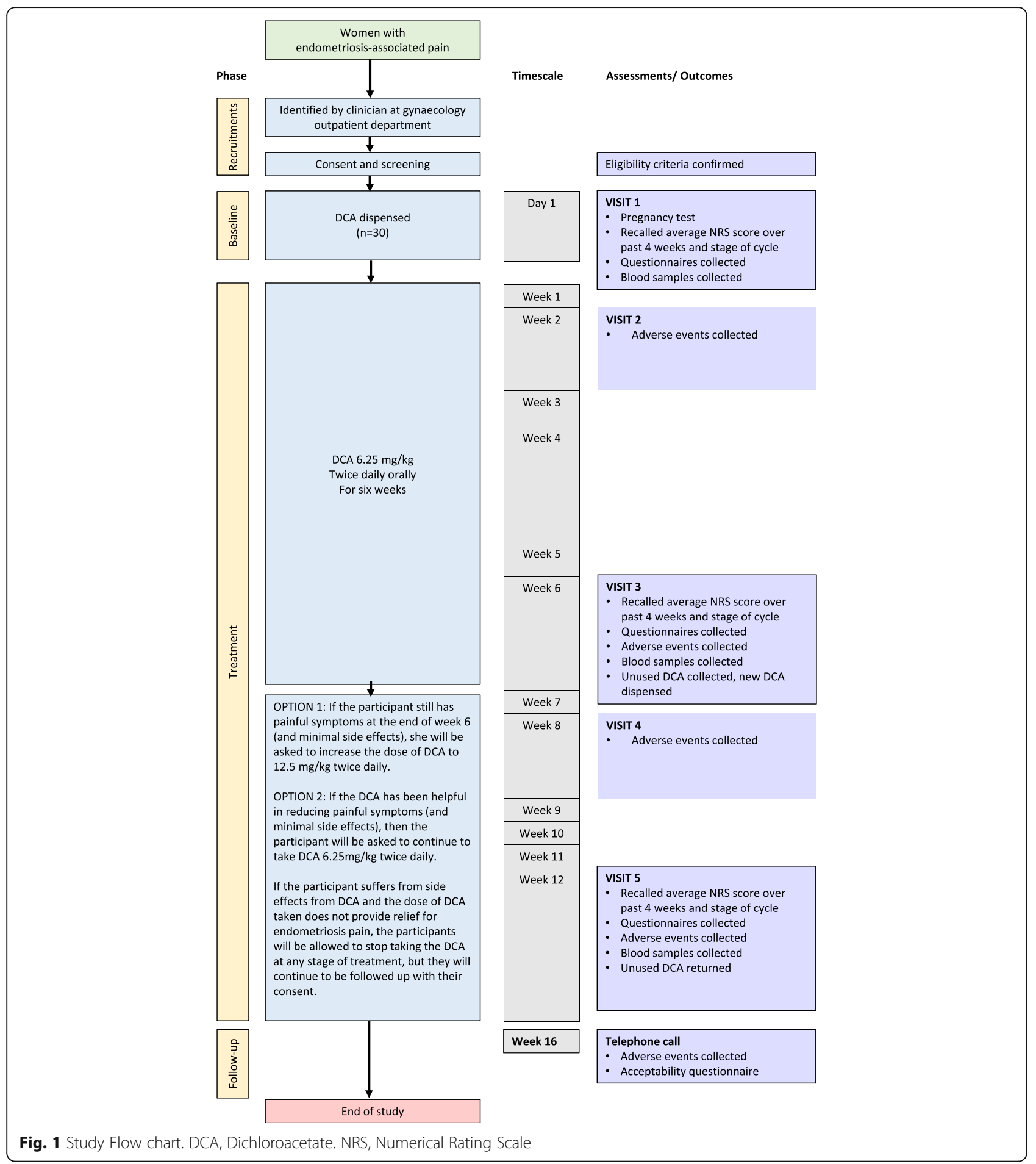

All questionnaires will be completed in private and anonymised. Patients will have a choice to complete them on paper during their visit or at home using an online link to secure REDCap database. Numerical Rating Scale (NRS) for pain will be collected on paper CRF only and will be recorded on the database that they were completed.

\section{Adverse events}

At each visit, participants will be asked about any adverse events (AEs) that have occurred during treatment. Any AEs that occur after joining the trial will be reported in detail in the participant's medical notes and $\mathrm{AE} \log$, and followed up until resolution of the event. All AEs will be assessed for seriousness, causality, 
expectedness and severity. All serious adverse events (SAEs) will be reported to the Academic and Clinical Central Office for Research and Development (ACCORD) Research Governance (http://www.accord.ed.ac. uk) and Quality Assurance Office based at the University of Edinburgh within $24 \mathrm{~h}$ of becoming aware that an SAE has occurred. Known side-effects of DCA (tingling or numbness, sleepiness, confusion, heartburn, mental fogginess and nausea) will not be recorded as AEs but will be recorded on the CRF at each visit. Admittance to hospital for surgery related endometriosis/pelvic pain, exacerbation of pain related to endometriosis/pelvic pain, or elective treatments planned prior to enrolment will not be reported as SAEs.

\section{Termination of study}

All participants will be required to stop DCA treatment after 12 weeks and return any remaining DCA to the investigator at the end of the study. Participants may withdraw from the trial at any point or a participant can be withdrawn by the investigator. Although pregnancy is not considered an adverse event, any participant's pregnancy will be recorded on a Pregnancy Notification Form and submitted to the ACCORD office within 14 days of being made aware of the pregnancy. All pregnant participants will be followed up until the outcome of the pregnancy. Data collection is envisaged to be completed in August 2020.

\section{Sample size}

The emphasis in this study is to establish feasibility, not statistical significance. This study is designed primarily to explore the effectiveness of the proposed field methodology: recruitment, retention, study processes and compliance with treatment. We will aim to recruit as many women as possible over a 6 -month period. We estimate that we will recruit 5-6 patients per month and will aim to recruit 30 patients. Data from this exploratory study will be used to refine sample size calculations for any future RCT.

\section{Statistical analysis}

Summary data will be used to describe baseline characteristics: mean and standard deviation, or median and interquartile range (IQR), and minimum, maximum for continuous variables and number (percentage) of individuals for categorical variables. Since this is a singlearm trial with no randomisation, there is no risk of patients not receiving their randomised treatment. However, patients will be assumed to have completed the treatment as prescribed in the primary analysis, unless they failed to start treatment.

\section{Primary endpoint}

The primary endpoint is to determine recruitment and retention rates. Using the information collected from the participant log, we will determine the number of patients recruited from the pool of eligible women and a $50 \%$ of 60, 95\% CI (36.8-63.2\%) recruitment will be deemed acceptable. While a retention rate of $100 \%$ would be ideal, we will consider a rate of $80 \%$ satisfactory. We will provide an estimate of the proportion and its 95\% CI (61.492.3\%). In addition, we will determine the nature and number of unanswered questions in each questionnaire. We aim to determine whether the trial design will perform well enough in the field to warrant rolling out the study to full trial. All analysis will be carried out in the University of Edinburgh.

\section{Secondary endpoints}

Acceptability of proposed methods of recruitment, follow-up, treatments and questionnaires used in this study will be assessed quantitatively using self-reported side-effects and acceptability questionnaires at the end of study. We also aim to determine if treatment is acceptable in terms of self-reported compliance (from treatment diaries). The blood samples collected from patients will be analysed at University of Edinburgh, QMRI, to detect the systemic levels of DCA to confirm participants' compliance. The assay will be developed in the research laboratory of the Edinburgh Clinical Research Facility. It will be analysed using mass spectrometry (choosing the instrument that will provide most accurate readings) and either Analyst MultiQuant software or Xcalibur Quantitate software. Descriptive statistics will be used to describe use of rescue analgesia, side-effects and compliance and will be compared by dose escalation or not.

\section{Data storage}

All consented participants will be allocated a trial participant number. A REDCap database will be used to record the data, entered by the member of the research team. The data from the database will be used for analysis of the trial and will be archived for a minimum of 15 years. The online questionnaires completed by patients will be directly linked to the REDCap database.

\section{Discussion}

Dichloroacetate (DCA) may be a potential novel nonhormonal treatment for endometriosis-associated pain in women. To date, this is the first study investigating DCA for endometriosis pain and assessing whether it is possible to achieve acceptable recruitment and retention rates. Given that many trials struggle with recruitment and often request extensions or become at risk of closure [23], investigating the feasibility and acceptability of 
treatments or interventions is of great importance to inform design of future definitive controlled trials [24]. If there are any indicators of problems with feasibility and acceptability affecting recruitment, retention, or safety, those relevant procedures will be revised and modified.

Our sample size of 30 women and 6 months recruitment period would be reasonable to provide a justified information of feasibility, acceptability to methodology (recruitment, retention, treatment, follow-up frequency), compliance rate and response to questionnaires. A key strength of the study is the clear definition of participant's disease as endometriosis diagnosed via laparoscopy within the past five years (excluding deep endometriosis), and the specific inclusion and exclusion criteria would be reflective to the patient's eligible to be on DCA in the future RCT based on their suitability for DCA as treatment option.

The questionnaires used to measure the physical and emotional functioning were selected based on the Initiative on Methods, Measurement, and Pain Assessment in Clinical Trials (IMMPACT) recommendations for chronic pain trials which would provide a reflective account on the women's experiences [15-18]. Although pain scores and satisfaction rates are being used as part of the study protocol, the trial is not designed nor powered for formal analysis of effectiveness. The questionnaire results (e.g. pain score, physical and emotional functioning) from this study will not be carried forward for future studies. However, the nature and number of unanswered questions in the questionnaire can help us to assess suitability of the questionnaires and identify important outcome measures for future study.

This study is limited to examining the feasibility and acceptability of DCA intervention within a single tertiary centre setting and does not examine the feasibility of a multi-centre trial. This study does not assess the willingness of participants to be randomised and therefore cannot estimate the recruitment rate of future RCT but allow estimation of number of eligible patients and estimation of time frame for recruitment and collection of data in future RCT. The results may not be truly representative of all patient demographics due to selection bias as participants are recruitment from a single centre and must be willing to comply with the frequent followup visits.

DCA offers promise for a novel, non-hormonal medical therapy for the treatment of endometriosisassociated pain. The success of this study will depend on developing appropriate recruitment strategies. Ideally, we would want to meet both our recruitment and retention targets before consideration of a larger randomised controlled trial. However, Covid-19 social distancing guidelines and the closure of many outpatient gynaecology clinics during the lockdown is making the collection of follow-up data more difficult as patients are less willing to attend non-urgent hospital appointments. Thus, our estimates of both recruitment and retention are likely to be underestimates of these figures under normal circumstances. Nonetheless, this study will still give us an estimate of the lower limit of these figures, which can then be used to inform a future clinical trial comparing the effectiveness of DCA to placebo administration for endometriosis-associated pain.

\section{Abbreviations \\ ACCORD: The academic and clinical central office for research and development; AEs: Adverse events; ASRM: American Society of Reproductive Medicine; BFI: Brief fatigue inventory; CRF: Case report form; CTIMP: Clinical trial of an investigational medicinal product; DCA: Dichloroacetate; \\ GCP: Good Clinical Practice; HPMC: Human peritoneal mesothelial cell; IMMP ACT: Initiative on methods, measurement, and pain assessment in clinical trials; IQR: Interquartile range; NHS: National Health Service; NRS: Numerical rating scale; PDK: Pyruvate dehydrogenase kinase; RCT: Randomised controlled trial; SAEs: Serious adverse events}

\section{Acknowledgements}

We are grateful to all the participants attending and staff working at NHS Lothian for their assistance in identifying potentially eligible participants.

\section{Authors' contributions}

LHRW, LW, PTKS, JD, AMD, MK, MCJ, GDF, YB and AWH developed the trial protocol and follow-up schedule. LW and JD chose the suitable statistical analysis for the data. HWL, AMD, MK, AWH and LHRW performed the literature review and were involved in drafting, editing and approval of final manuscript. All authors read and approved the final manuscript. AWH is the chief investigator for the study.

\section{Funding}

This study is funded by the MRC confidence in Concept Scheme (MRC/CIC6/ 58) and an MRC Centre Grant (MRC G1002033). The funder has had no role in the design of the study and will have no role in the collection, analysis and interpretation of data and in writing the manuscript.

\section{Availability of data and materials}

Data sharing is not applicable to this article as no datasets were generated or analysed during the current study.

\section{Ethics approval and consent to participate}

The study will be conducted in accordance with the principles of the International Conference on Harmonisation Tripartite Guideline for Good Clinical Practice (ICH GCP). Ethical approval was obtained from Scotland A Research Ethics Committee (19/SS/0063). Consent to participate will be required from all participants.

Scotland A Research Ethics Committee (NHREC 19/SS/0063)

\section{Consent for publication}

Not applicable.

\section{Competing interests}

AWH receives grant funding from the NIHR, MRC, Chief Scientist's Office, Wellcome Trust, Wellbeing of Women, Ferring and Roche. He has received honoraria for consultancy for Ferring, Roche Diagnostics, Nordic Pharma and Abbvie. HWL, LHRW, MK, AMD, LW, PTKS, JD, MCJ, YB and GDF declare no competing interests.

\section{Author details}

'MRC Centre for Reproductive Health, Queen's Medical Research Institute, University of Edinburgh, 47 Little France Crescent, Edinburgh EH16 4TJ, UK. ${ }^{2}$ Usher Institute, NINE Edinburgh BioQuarter, 9 Little France Road, Edinburgh EH16 4UX, UK. ${ }^{3}$ Centre for Inflammation Research, Queen's Medical Research Institue, University of Edinburgh, Edinburgh EH16 4TJ, UK. ${ }^{4}$ Clinical Trials Unit, University of Nottingham, University Park, Nottingham NG7 2RD, UK.

${ }^{5}$ Institute of Clinical Sciences, University of Birmingham, Edgbaston, 
Birmingham B15 2TT, UK. 'Reproductive Medicine and Maternal Health, Ferring Research Institute, San Diego, CA 92121, USA.

Received: 20 April 2020 Accepted: 15 February 2021

Published online: 12 March 2021

\section{References}

1. Horne AW, Saunders PTK. SnapShot: endometriosis. Cell. 2019;179(7):1677-.e1.

2. Johnson NP, Hummelshoj L. Consensus on current management of endometriosis. Hum Reprod. 2013;28(6):1552-68.

3. Kuznetsov L, Dworzynski K, Davies M, Overton C. Diagnosis and management of endometriosis: summary of NICE guidance. Bmj. 2017;358: j3935.

4. Yap C, Furness S, Farquhar C. Pre and post operative medical therapy for endometriosis surgery. 2004.

5. Simoens S, Dunselman G, Dirksen C, Hummelshoj L, Bokor A, Brandes I, et al. The burden of endometriosis: costs and quality of life of women with endometriosis and treated in referral centres. Hum Reprod. 2012;27(5):1292-9.

6. Saraswat L, Ayansina D, Cooper KG, Bhattacharya S, Horne AW, Bhattacharya S. Impact of endometriosis on risk of further gynaecological surgery and cancer: a national cohort study. Bjog. 2018;125(1):64-72.

7. Guo S-W. Recurrence of endometriosis and its control. Human Reproduction Update. 2009;15(4):441-61.

8. Horne AW, Saunders PTK, Abokhrais IM, Hogg L. Top ten endometriosis research priorities in the UK and Ireland. Lancet. 2017;389(10085):2191-2.

9. Anglesio MS, Papadopoulos N, Ayhan A, Nazeran TM, Noe M, Horlings HM et al. Cancer-associated mutations in endometriosis without cancer. N Engl J Med. 2017:376(19):1835-48.

10. Young VJ, Ahmad SF, Duncan WC, Horne AW. The role of TGF- $\beta$ in the pathophysiology of peritoneal endometriosis. Hum Reprod Update. 2017; 23(5):548-59.

11. Young VJ, Brown JK, Maybin J, Saunders PT, Duncan WC, Horne AW. Transforming growth factor-beta induced Warburg-like metabolic reprogramming may underpin the development of peritoneal endometriosis. J Clin Endocrinol Metab. 2014;99(9):3450-9.

12. Young VJ, Brown JK, Saunders PT, Duncan WC, Horne AW. The peritoneum is both a source and target of TGF-beta in women with endometriosis. PloS One. 2014;9(9):e106773.

13. Hirschhaeuser F, Sattler UGA, Mueller-Klieser W. Lactate: a metabolic key player in cancer. Cancer Res. 2011;71(22):6921-5.

14. Horne AW, Ahmad SF, Carter R, Simitsidellis I, Greaves E, Hogg C, et al. Repurposing dichloroacetate for the treatment of women with endometriosis. Proc Natl Acad Sci. 2019:116(51):25389-91.

15. Haugrud AB, Zhuang Y, Coppock JD, Miskimins WK. Dichloroacetate enhances apoptotic cell death via oxidative damage and attenuates lactate production in metformin-treated breast cancer cells. Breast Cancer Res Treat. 2014;147(3):539-50.

16. Chu QS, Sangha R, Spratlin J, Vos LJ, Mackey JR, McEwan AJ, et al. A phase I open-labeled, single-arm, dose-escalation, study of dichloroacetate (DCA) in patients with advanced solid tumors. Investig New Drugs. 2015;33(3):603-10.

17. Michelakis ED, Gurtu V, Webster L, Barnes G, Watson G, Howard L, et al. Inhibition of pyruvate dehydrogenase kinase improves pulmonary arterial hypertension in genetically susceptible patients. Sci Transl Med. 2017;9(413): eaao4583. https://doi.org/10.1126/scitranslmed.aao4583.

18. Michelakis ED, Sutendra G, Dromparis P, Webster L, Haromy A, Niven E, et al. Metabolic modulation of glioblastoma with dichloroacetate. Sci Transl Med. 2010;2;(31):31ra4

19. Jones $\mathrm{G}$, Jenkinson C, Taylor N, Mills A, Kennedy S. Measuring quality of life in women with endometriosis: tests of data quality, score reliability, response rate and scaling assumptions of the Endometriosis Health Profile Questionnaire. Hum Reprod. 2006;21(10):2686-93.

20. Freynhagen $\mathrm{R}$, Baron $\mathrm{R}$, Gockel U, Tolle TR. painDETECT: a new screening questionnaire to identify neuropathic components in patients with back pain. Curr Med Res Opin. 2006;22(10):1911-20.

21. Mendoza TR, Wang XS, Cleeland CS, Morrissey M, Johnson BA, Wendt JK et al. The rapid assessment of fatigue severity in cancer patients: use of the Brief Fatigue Inventory. Cancer. 1999;85(5):1186-96.

22. Sullivan MJL, Bishop SR, Pivik J. The Pain Catastrophizing Scale: Development and Validation. Vol. 7, Psychological Assessment. 1995.
23. Blatch-Jones AJ, Pek W, Kirkpatrick E, Ashton-Key M. Role of feasibility and pilot studies in randomised controlled trials: a cross-sectional study. BM Open. 2018:8(9):e022233.

24. Eldridge SM, Lancaster GA, Campbell MJ, Thabane L, Hopewell S, Coleman $C L$, et al. Defining feasibility and pilot studies in preparation for randomised controlled trials: development of a conceptual framework. PloS One. 2016; 11(3):e0150205

\section{Publisher's Note}

Springer Nature remains neutral with regard to jurisdictional claims in published maps and institutional affiliations.
Ready to submit your research? Choose BMC and benefit from:

- fast, convenient online submission

- thorough peer review by experienced researchers in your field

- rapid publication on acceptance

- support for research data, including large and complex data types

- gold Open Access which fosters wider collaboration and increased citations

- maximum visibility for your research: over $100 \mathrm{M}$ website views per year

At $\mathrm{BMC}$, research is always in progress.

Learn more biomedcentral.com/submissions 